\title{
Apoio matricial em saúde mental entre CAPS e Saúde da Família: trilhando caminhos possíveis
}

\author{
Fabiane Minozzo - Universidade de Brasilia, Brasilia/DF, Brasil \\ Ileno Irídio da Costa - Universidade de Brasilia, Brasilia/DF, Brasil
}

\begin{abstract}
Resumo
Neste artigo discute-se a experiência de implantação do apoio matricial em saúde mental, prática que visa ampliar a resolubilidade e produzir maior responsabilização pelas situações de saúde mental na Saúde da Família (SF). Objetiva-se analisar a implantação do apoio matricial entre CAPS III e equipes de SF. Trata-se de uma pesquisa-ação cujos instrumentos foram os grupos operativos de reflexão e a resposta a questionários. Realizaram-se grupos com profissionais da SF, do CAPS e dos dois serviços conjuntamente. No que tange à SF, os resultados apontaram dificuldades na abordagem dos casos de saúde mental e a coexistência dos modos asilar e psicossocial nas práticas de cuidado. Em relação ao CAPS, indicaram a importância do fortalecimento do trabalho em equipe. Conclui-se que há necessidade de fortalecer a saúde mental na atenção primária à saúde, com investimentos na educação permanente, no estabelecimento de indicadores e na integração entre CAPS e SF.

Palavras-chave: Serviços de Saúde Mental, Atenção primária à saúde, Apoio matricial, Centro de Atenção Psicossocial (CAPS).
\end{abstract}

\section{Matrix support in mental health between Psychosocial Attention Center (CAPS) and Family Health Teams: analyzing possible paths}

\begin{abstract}
This paper discusses the experience of implementing the matrix support in mental health practice that aims to produce higher resolution and accountability for mental health situations in Family Health. The objective of the present study is to analyze the implementation of the mental health "matrix support" practice among Psychosocial Attention Centers (CAPS III) and Family Health Teams. This is an action-research that had as instruments operational groups of reflection and the response to questionnaires. Groups were held with Family Health professionals, with CAPS professionals and with professionals from the two services together. Regarding the Family Health services, the result indicated difficulties in addressing mental health cases and the coexistence of sheltering and psychosocial models of care practice in their work. In relation to the CAPS, they indicated the importance of strengthening teamwork to achieve the "matrix support" practice. The results indicate the need to strengthen mental health services within the primary health care, by investing in continuing education, establishing indicators and integrating the CAPS and Family Health services.

Keywords: Mental health services, primary Health care, Matrix support, Psychosocial Attention Center (CAPS).
\end{abstract}

\section{Apoyo matricial de salud mental entre CAPS y Salud de la Familia: trillando caminos posibles}

\begin{abstract}
Resumen
En este trabajo se analiza la experiencia de la aplicación de la matriz de soporte en la práctica de salud mental que tiene como objetivo producir una resolución más alta y la responsabilidad de la salud mental en situaciones de Salud de la Familia. El objetivo es analizar la aplicación de la matriz de soporte en salud mental entre los CAPS III y los Equipos de Salud de la Familia (SF). Ese es un grupo de investigación-acción cuyos instrumentos estaban operando la reflexión y la respuesta a los cuestionarios. Había grupos con los profesionales de ciencia ficción, los CAPS y de los dos servicios juntos. En cuanto a la SF, los resultados indicaron dificultades para hacer frente a los casos de salud mental y la convivencia del asilo y de los modelos psicosociales de las prácticas de cuidado. En relación a los CAPS, señalaron la importancia de fortalecer el trabajo en equipo para lograr el apoyo de la matriz. Se concluyó que hay la necesidad de fortalecer la salud mental en atención primaria, con inversiones en la educación permanente, el establecimiento de indicadores y la integración entre los CAPS y SF.

Palabras-clave: Servicios de salud mental, Atención primaria de salud, Matriz de soporte, Centro de Atención Psicosocial (CAPS).
\end{abstract}

A ideia central deste artigo é discutir a implantação do apoio matricial em saúde mental dos Centros de Atenção Psicossocial (CAPS) às equipes de Saúde da Família (SF). O apoio matricial objetiva aumentar a resolubilidade da SF e produzir maior responsabilização pela saúde mental na Atenção Primária à Saúde (APS). Busca, com isso, romper com a lógica dos encaminhamentos indiscriminados, ampliar a clínica e auxiliar as equipes a trabalhar a dimensão do sofrimento psíquico.
O apoio matricial é um arranjo formulado por Campos (1999), configurando-se como um suporte técnico especializado ofertado às equipes de APS a fim de ampliar seu campo de atuação e qualificar suas ações, podendo ser realizado por profissionais de diversas áreas especializadas. No caso deste artigo, aborda-se o apoio matricial em saúde mental, oferecido pelos profissionais dos CAPS. Tal prática é consoante à Política Nacional de Saúde Mental, que prioriza a implantação de serviços e ações de saúde mental de base comunitária, capazes de atender com 
resolubilidade os pacientes que necessitem de atenção (Brasil, 2005). Os CAPS e o fortalecimento da APS em ações de saúde mental são fundamentais na atenção psicossocial.

A atenção psicossocial é uma expressão que indica a necessidade de se construirem oportunidades para as pessoas em sofrimento psíquico, para que possam exercer sua cidadania e atingir seu potencial de autonomia no território em que vivem. A atenção psicossocial tem como objeto de trabalho a existência de sofrimento em relação ao corpo social, e como objetivo, a emancipação social do sujeito (Brasil, 2010; Costa-Rosa, 2000).

Os CAPS são considerados serviços estratégicos da Reforma Psiquiátrica brasileira, apontando para a possibilidade de organização de uma rede substitutiva ao hospital psiquiátrico no país. Existem diferentes tipos de CAPS, diferenciados de acordo com o porte, capacidade de atendimento, clientela atendida e perfil populacional dos municípios brasileiros. Os CAPS III, em foco neste estudo, são serviços de maior complexidade, com funcionamento 24 horas, durante todos os dias, inclusive feriados e finais de semana. $\mathrm{Na}$ rede de saúde mental, os CAPS III são importantes pela possibilidade de acolher à crise, oferecer acolhimento noturno, proporcionar atenção integral e evitar internações psiquiátricas (Brasil, 2004).

Já os serviços de APS se constituem na porta de entrada preferencial do Sistema Único de Saúde (SUS), local onde são identificadas e coordenadas as respostas para as necessidades de saúde das pessoas, suas famílias e comunidades. Starfield (1994) aponta que, em sua forma mais altamente desenvolvida, a APS é responsável pela resolução de cerca de $85 \%$ dos problemas de saúde da população.

No Brasil, de acordo com a Portaria $n^{\circ}$ 2.488/2011, de 21 de outubro, a Saúde da Família (SF), estratégia prioritária de reorganização da APS, é responsável por um dos mais significativos movimentos de ampliação do acesso à saúde. A Estratégia Saúde da Família (ESF) é operacionalizada mediante a implantação de equipes multiprofissionais, compostas por médico, enfermeiro, técnico de enfermagem e agentes comunitários de saúde (ACS). Tais equipes atuam em unidades básicas de saúde e possuem a responsabilidade de prestar atenção à saúde a uma população adscrita, tendo como máximo recomendado o equivalente a quatro mil pessoas sob sua responsabilidade.

No que se refere à saúde mental, identifica-se que é necessário investir em estratégias que incluam ações voltadas a tal área no cotidiano da ESF. Para tal, o apoio matricial em saúde mental é considerado uma prática fundamental, pois auxilia na condução de casos e situações complexas, bem como apoia o planejamento de ações e a educação permanente das equipes.

Este artigo aborda resultados de pesquisa que teve como objetivo analisar o processo de implantação do apoio matricial em saúde mental de um CAPS III às equipes de SF, em uma comunidade, no município do Rio de Janeiro, Brasil. Para tal, tomou como objetivos específicos: a) investigar o processo de implantação do apoio matricial em saúde mental do CAPS III às equipes de SF; b) identificar as principais facilidades e dificuldades no processo de implantação do apoio matricial em saúde mental; c) promover aos participantes da pesquisa a reflexão sobre o apoio matricial em saúde mental; e d) identificar as principais ações de apoio matricial viáveis de realização ou qualificação.

Ressalta-se que os serviços pesquisados foram implantados no ano de 2010. O CAPS III e a Unidade de SF analisados atuam na mesma unidade física, mas cada serviço possui suas salas e ambientes próprios, as equipes de SF atuando na parte térrea e o CAPS no segundo andar.

\section{Metodologia}

Trata-se de uma pesquisa qualitativa, de cunho exploratório, estruturada na modalidade da pesquisaação. Por seu duplo objetivo de modificar a realidade e produzir conhecimentos, a pesquisa-ação favorece que os participantes e pesquisadores se envolvam de modo cooperativo e participativo, produzindo novas reflexões e práticas (Barbier, 2002; Thiollent, 1996).

Esta pesquisa teve como instrumentos os grupos operativos de reflexão e a resposta a questionários. Foram realizados grupos com profissionais da SF, do CAPS e dos dois serviços conjuntamente. As técnicas utilizadas foram construídas conjuntamente entre pesquisadores e participantes, optando-se por leitura e debate de textos, discussão sobre experiências de apoio matricial e reflexões em grupo. Os encontros dos grupos foram coordenados pela pesquisadora, primeira autora deste trabalho, gravados em áudio e subsequentemente transcritos.

Os questionários foram compostos por cinco perguntas, quatro abertas e uma fechada, elaborados para a pesquisa em tela, respondidos pelos participantes no início e no final da investigação. Estes buscaram identificar o conceito de apoio matricial dos participantes, suas experiências de matriciamento, suas dúvidas e percepções sobre as facilidades e dificuldades para a implantação desta prática.

Foram realizados três encontros de grupo com os profissionais da SF, três com os profissionais do CAPS 
e três integrando os dois serviços. Participaram 15 profissionais do CAPS III e 6 profissionais de nível superior da Saúde da Família. Optou-se por restringir a participação na pesquisa aos profissionais de nível superior da ESF por entender-se que os mesmos poderiam discutir o tema apoio matricial com os demais integrantes da equipe de $\mathrm{SF}$, como os agentes comunitários de saúde. Os grupos ocorreram no período de novembro de 2010 a fevereiro de 2011.

Utilizou-se a análise de conteúdo e sua técnica temática como forma de tratamento das informações. Segundo Bardin (1977), a análise de conteúdo consiste em um conjunto de técnicas de análises de comunicações, utilizando-se de procedimentos sistemáticos e objetivos de descrição do conteúdo das mensagens. Para a análise de conteúdo realizou-se a pré-análise, na qual se fez a escolha do material coletado e a leitura flutuante. Foram utilizadas as transcrições dos grupos $\mathrm{e}$ as respostas aos questionários. Após, foi realizada a categorização, com os temas que tiveram a mesma significação (Franco, 2008). Para identificar os temas mais recorrentes foi utilizada a análise quantitativa. Verificou-se a quantidade de unidades de registro - menções nas falas e respostas dos participantes aos questionários - e contabilizada a frequência em cada tema. A pesquisa foi submetida e aprovada pelo Comitê de Ética em Pesquisa da Secretaria Municipal de Saúde e Defesa Civil do Rio de Janeiro.

\section{Análise e Discussão}

A fim de sistematizar a discussão dos resultados, serão apresentados os principais achados em cada grupo pesquisado.

\section{A voz da Saúde da Família}

Este grupo foi composto pelos profissionais da ESF que participaram do estudo nos três primeiros encontros dos grupos operativos de reflexão e responderam aos questionários desta pesquisa. Destacaram-se duas categorias analíticas: a) Saúde Mental na ESF; e b) Apoio Matricial em Saúde Mental: perspectiva da SF.

A categoria Saúde Mental na Estratégia Saúde da Família obteve setenta e três menções, evidencia as dificuldades referidas pelos profissionais da SF na atenção em saúde mental e a coexistência dos dois modelos, asilar e clínica ampliada, nas ideias e práticas de cuidado da SF no que se refere à saúde mental.

Foram elucidadas dificuldades dos profissionais da ESF no que se refere à atenção em saúde mental, principalmente na abordagem dos casos e das situações que envolvem práticas que exigem mais que o saber biomédico, o que foi associado com falta de conhecimento e insuficiências na formação. "A gente não está capacitado pra resolver os pequenos problemas de saúde mental ainda" (Dentista); "Eu acho que a graduação deixa muito a desejar. A parte prática era de ficar olhando e não fazendo nada [...]. Mas na aula não teve nada muito aprofundado mesmo" (Enfermeira).

O medo do desconhecido, a insegurança na abordagem dos casos de saúde mental, o preconceito dos profissionais em relação aos pacientes foram identificados no conteúdo das falas dos participantes da SF pesquisados. Diante dessa realidade, alguns portadores de sofrimento psíquico não são identificados e outros são ignorados no cotidiano das equipes SF. "A gente que não identifica, ou não quer identificar" (Dentista); "Mas eu acho que nós também temos um preconceito" (Enfermeira).

Tais achados são consoantes ao referido por Onocko Campos (2010), de que falta preparo dos profissionais na APS para o desenvolvimento das práticas em saúde mental. As ações desenvolvidas ainda estão vinculadas ao modelo biomédico, acarretando uma "série de posturas inadequadas, como práticas discriminatórias e preconceituosas com relação ao portador de sofrimento psíquico, ações moralistas, normatizadoras e repressivas, infantilização" (p. 227).

Nessa categoria, destaca-se também a coexistência dos dois modos de atenção, asilar e clínica ampliada, no cuidado em saúde mental promovido pelas equipes de SF. De acordo com Costa-Rosa (2000), o modo asilar centra o cuidado nos aspectos orgânicos, no diagnóstico e no medicamento. A clínica ampliada, por sua vez, "traduz-se numa ampliação do objeto de trabalho e na busca de resultados eficientes, com inclusão de novos instrumentos" (Brasil, 2009, p. 26). É uma prática que parte do "núcleo biomédico para os aspectos subjetivos e sociais de cada sujeito, respeitando a característica singular de cada caso [...] sem abrir mão de critérios técnicos previamente definidos" (Campos \& Guerreiro, 2010, p. 138).

Identificaram-se nos profissionais da SF concepções que apontam para o reconhecimento da importância do vínculo e a valoração da escuta e da troca entre os usuários como promotores de saúde mental, aspectos da clínica ampliada.

Por isso é bom esse vínculo, da gente estar participando mais do cotidiano, pra saber quem é quem, de que maneira abordar, que tipo de abordagem e ter paciencia, porque estabelecer vinculo demanda paciência. (Enfermeira)

Mas, nas situações de crise em saúde mental ou no atendimento aos casos mais graves, os profissionais da ESF relataram ações baseadas no modo asilar. O 
encaminhamento para a internação psiquiátrica, a imposição do medicamento e a falta de contratualização com os pacientes e familiares foram algumas das ações referidas em tais situações. "Eu mesma ia chamar a ambulância para que ele pudesse ser levado pra emergência psiquiátrica, porque como que eu vou deixar ele sair [...]. Ele não quer ir, eu estou mandando ele ir". (Enfermeira); "Se tivesse uma medicação que pudesse controlar esse comportamento dele, eu faria" (Dentista).

Já a categoria Apoio Matricial em Saúde Mental: perspectiva da SF, com sessenta e seis unidades de registro, sinaliza a concepção das equipes de SF sobre apoio matricial, as expectativas dos participantes em relação ao tema e o estado de arte dessa prática nas equipes de $\mathrm{SF}$ pesquisadas.

Ao iniciar a pesquisa, identificou-se que os profissionais da ESF desconheciam o conceito e a prática do apoio matricial. Dos seis participantes da ESF, cinco referiram desconhecer o tema. Ao final da investigação, o conceito de apoio matricial estava mais claro para os profissionais da ESF.

As expectativas dos profissionais da ESF em relação ao apoio matricial giram em torno de receber auxílio para a condução da prática clínica.

O que espero em relação ao matriciamento é saber como proceder com os pacientes com problemas mentais, de que forma é melhor tratá-los, em que momento a gente deve intervir $[. .$.$] e se tem que encaminhar para um$ apoio especializado. (Dentista)

Em alguns momentos, as expectativas delegaram grande poder aos profissionais do CAPS, como se eles soubessem de todas as respostas da saúde mental. Ainda, os profissionais da ESF demonstraram não ter clareza da especificidade do CAPS III. "Um paciente com Síndrome de Down, ou pacientes com retardo mental, eles são atendidos no CAPS?" (Dentista); "O que falta é a gente conhecer mesmo. Você tem que procurar, eu cheguei lá meio perdida [no CAPS], saí procurando qualquer pessoa" (Enfermeira).

Dentre as dificuldades para a implantação do apoio matricial, a partir da ESF, destacarou-se a rotina intensa no cotidiano, que foi associada à excessiva carga de trabalho, gerando indisponibilidade de tempo e de investimentos em outras ações diferentes das já desenvolvidas. Outro aspecto mencionado foi a dificuldade de adesão dos profissionais ao apoio matricial. "Uma das dificuldades é de convencer os demais profissionais na unidade da importância da participação no processo de matriciamento". (Enfermeira). Dentre as facilidades de implantação do apoio matricial, foi mencionada a proximidade entre os dois serviços, por atuarem na mesma estrutura física, e a vontade e empenho das equipes de $\mathrm{SF}$ em prestar atenção em saúde de qualidade aos usuários.

$\mathrm{Na}$ perspectiva da ESF, o processo de implantação do apoio matricial é incipiente, não ocorre com frequência e regularidade. As ações ocorrem quando algum caso clínico atendido pelos dois serviços - CAPS e ESF - demanda intervenções conjuntas. Sendo assim, identificou-se que os encontros deste estudo incitaram a reflexão e maior apropriação do conceito "apoio matricial" por parte da SF, bem como despertaram o desejo dos participantes em fortalecer esta prática.

O que dizem os profissionais do CAPS III?

Este grupo foi composto pelos profissionais do CAPS III que participaram dos três primeiros encontros da pesquisa. Identificaram-se as seguintes categorias: a) Trabalho em Equipe e; b) Apoio Matricial em Saúde Mental: perspectiva do CAPS.

O Trabalho em Equipe no CAPS, com quarenta e sete menções, foi apontado pelos participantes como um dos fatores que facilita, fortalece e traz maior uniformidade para a realização do apoio matricial. As posições tomadas em equipe foram citadas como importantes para a condução do matriciamento, porém, a pesquisa evidenciou que os profissionais têm dificuldades para se reunir e discutir essa prática. A falta de tempo no trabalho foi apontada como um dos obstáculos para a realização da discussão interna sobre apoio matricial. $O$ que pode ser identificado nas seguintes falas:

A gente tem que discutir matriciamento, os textos, mas também é importante a gente discutir isso dentro do processo de trabalho [...]. A gente precisa olhar pra gente e cavar um tempo, apesar de toda a correria, apesar das agendas. (Nutricionista)

Porque às vezes a gente trabalha, trabalha, fica muito cansado e pra onde vai esse trabalho? É a gente ter condiçoes, vestir a camisa do nosso serviço e não sair falando e fazendo besteira. (Psicóloga)

Foram identificados também conflitos interpessoais, falta de comunicação e de articulação e, consequentemente, prejuízo ao desenvolvimento do apoio matricial e sofrimento do trabalhador da saúde mental. Foi percebido o trabalho isolado de cada especialidade, gerando falta de integração e de troca entre os profissionais: "A gente não se comunica, é o meu trabalho, é o meu serviço, sou eu que faço" (Enfermeira).

As pessoas não perguntam, nem querem saber o que você está fazendo e quando você apresenta também não querem saber. Então, eu acho que essa é uma questão de adoecimento de uma equipe em saúde mental. (Psicóloga) 
Outro aspecto destacado diz respeito aos espaços de reuniões de equipe semanais, àquelas em que estão presentes a maioria dos integrantes do serviço. A falta de objetividade, o julgamento, a dificuldade de escuta entre os colegas e a pouca circulação da palavra apareceram como elementos da reunião, despotencializando a comunicação e as trocas profissionais.

Geralmente, na reunião de equipe [...] a própria equipe não dá oportunidade de você falar. As vezes você vai falar uma coisa e as outras pessoas falam outra, parece que o que você disse não tem nada a ver. (Técnico de Enfermagem)

O trabalho em equipe é considerado determinante para o rompimento das práticas asilares, atuando como uma das formas de enfrentar a fragmentação do trabalho e do sujeito, rumo à integralidade do cuidado. Nesse sentido, a construção do novo modelo de atenção em saúde mental pressupõe uma atuação mais plural e integral por parte dos profissionais e uma multiplicidade de enfoques. Dessa forma, "a equipe é um dos atores principais dessa configuração" (Guljor, 2003, p. 85).

Este estudo sinaliza a necessidade de fortalecer o trabalho em equipe no CAPS, potencializando espaços como o da reunião de equipe, para que a palavra possa circular entre todos os integrantes, a fim de que haja reflexão sobre o trabalho coletivo e para que os conflitos existentes possam emergir e ser trabalhados. Entende-se que o trabalho em equipe é fundamental para que o apoio matricial se efetive na lógica da atenção psicossocial.

A categoria Apoio Matricial: perspectiva do CAPS, com cinquenta e uma menções, demonstrou, de forma semelhante ao grupo da ESF, que muitos participantes no início do estudo desconheciam o conceito de apoio matricial. No primeiro encontro da investigação, sete dos quinze participantes referiram não saber o que é apoio matricial, situação modificada no último encontro da pesquisa, em que o conceito estava mais claro entre todos os participantes.

Ao ser investigada a experiência profissional antes do ingresso no CAPS, no que se refere ao matriciamento, constatou-se que somente três dos quinze investigados, tinham experiências prévias. O desconhecimento conceitual e a pouca vivência de matriciamento refletem em insegurança na realização do apoio matricial. Nesse sentido, identificou-se a necessidade da equipe do CAPS sentir-se autorizada para se reunir com a ESF, diminuindo a cobrança sobre a necessidade de conhecer tudo o que se refere ao apoio matricial e concebendo o matriciamento como construção conjunta, compartilhamento e ampliação do olhar de todos os envolvidos e não como uma transmissão vertical do conhecimento.

Assim, na perspectiva do CAPS, a implantação do apoio matricial às equipes estudadas está em processo inicial, corroborando os dados encontrados no grupo da ESF. Os participantes identificaram a necessidade de estreitar os laços entre si, como equipe, e com a ESF fomentando a integração entre os serviços. Da mesma forma que na ESF, acredita-se que os encontros da pesquisa possibilitaram maior apropriação do conceito de apoio matricial e fomentaram o início de alguns encontros de matriciamento.

\section{A integração Saúde da Família e CAPS}

A análise apresentada a seguir diz respeito aos resultados produzidos nos três encontros de integração entre os dois serviços, CAPS III e ESF, formando um grupo misto. Identificaram-se as seguintes categorias: a) Inserção do apoio matricial no processo de trabalho da ESF: um desafio e; b) Apoio matricial em saúde mental: trilhando caminhos possíveis.

A categoria "Inserção do apoio matricial no processo de trabalho da Estratégia Saúde da Família: um desafio", com setenta e nove unidades de registro, sinaliza as dificuldades no processo de trabalho da ESF que obstam a implantação do apoio matricial em saúde mental. Ainda, apresenta os desafios a serem enfrentados para a realização do matriciamento, bem como as reflexões do grupo perante a visualização de tais obstáculos.

Dentre as dificuldades elucidadas, destacou-se a pressão dos profissionais da ESF para o alcance de metas, que são entendidas como cobranças para a obtenção de uma produção numérica de atendimentos e de outras atividades. Os relatos apontaram para a sensação de pressão, culminando em problemas de relacionamento interpessoal. "É porque nós vivemos numa pressão muito grande, às vezes a gente tem problemas de relacionamento dos profissionais. Problemas mesmo por conta da demanda, da pressão" (Enfermeira).

A sensação de pressão e o tempo reduzido para os atendimentos geram nos profissionais da ESF dificuldade de escuta dos aspectos subjetivos, importante para a integralidade do cuidado. Além disso, causam frustração, pois os profissionais referem não realizar algumas atividades com qualidade.

A gente sofre muito, eu acabo sem tempo de promover saúde. É uma coisa que me angustia muito. Então, a demanda é intensa por atendimento [...]. Neste momento, a gente tá meio sufocado. (Dentista)

Neste sentido, o grupo discutiu sobre a importância da ESF dispor de mais tempo para atender os sujeitos em sofrimento psíquico, objetivando a 
escuta das angústias, o entendimento da história e do momento de vida de cada paciente, o que possibilita traçar estratégias de intervenção condizentes com a singularidade de cada caso.

Para subsidiar esta discussão foram consultados documentos da Secretaria Municipal de Saúde e Defesa Civil do Rio de Janeiro (2009). A partir da verificação, identificou-se que as metas propostas estão fundamentadas em parâmetros, estudos e pesquisas nacionais e a proposta de atividades semanais, para os profissionais de nível superior da ESF, se baseia em consultas, visitas domiciliares, atividades em grupo, de promoção de saúde, reuniões de equipe e interconsulta ou telemedicina. No que se refere ao tempo de atendimento, é proposta uma média de vinte minutos para as consultas médicas e de vinte e cinco e trinta minutos para os atendimentos de enfermagem e de odontologia, respectivamente. Levando-se em consideração que alguns atendimentos da ESF podem ser mais ágeis, demandando menos que a média de tempo proposta, que as famílias são acompanhadas continua e longitudinalmente, tanto nas consultas individuais, quanto no território, identifica-se que o processo de trabalho nas equipes pesquisadas necessita ser refletido e discutido, possibilitando, assim, que o aspecto tempo seja debatido e estratégias sejam traçadas, evitando-se que seja um impeditivo para a realização do apoio matricial.

O trabalho dos ACS consistiu em tema de destaque na discussão do grupo misto. No contexto da implantação do apoio matricial e da consequente responsabilização da ESF pelos casos de saúde mental do território, os ACS têm papel fundamental. Mas, na percepção dos participantes da pesquisa, esses profissionais estão se sentindo sobrecarregados.

Eles estão sofrendo a mesma pressão, [...] eles conversam com a gente, insatisfeitos com muitas coisas, sobrecarregados, e ai gera problema na equipe, briga na equipe, brigas maiores, então tá mesmo turbulento. (Dentista)

Ao ser pensada a inclusão do ACS nas discussões sobre matriciamento e seu papel com a saúde mental, destacou-se a necessidade de construção de estratégias que desconstruam a ideia de que o apoio matricial é mais uma tarefa a dar conta. De fato, o apoio matricial consiste em mais uma ação na ESF, mas a troca entre os serviços e o compartilhamento dos casos difíceis podem propiciar qualidade e leveza ao trabalho.

Por meio das reflexões proporcionadas pelos encontros da pesquisa, os profissionais dos dois serviços construíram direcionamentos para a implantação do apoio matricial. O grupo identificou que o matriciamento relaciona-se com o compartilhamento da prática, com a coresponsabilização pelos casos clínicos e com o processo de educação permanente, favorecendo a aprendizagem tanto da ESF quanto dos profissionais do CAPS.

Além disso, o grupo discutiu sobre o cuidado oferecido aos cuidadores, destacando que o apoio matricial não contempla espaços de psicoterapia para os trabalhadores e sim momentos de escuta que fortaleçam as equipes para lidar com a complexidade das situações e casos de sofrimento psíquico.

Esses espacos não devem se configurar como psicoterapia para os profissionais/equipes, mas sim como momentos de trocas, construções mútuas que visam auxiliar na condução de casos e das situações complexas vividas no cotidiano do trabalho. (Brasil, 2010, p. 43)

A categoria "Apoio matricial em saúde mental: trilhando caminhos possíveis", com sessenta e cinco unidades de registro, apresenta temas relacionados à organização das agendas de matriciamento e aos encaminhamentos dos casos de saúde mental da ESF para o CAPS. Contempla, ainda, a identificação das ações de apoio matricial possíveis de serem realizadas.

No que tange à organização das agendas conjuntas, os integrantes demonstraram dificuldades em propor agendas de matriciamento, apresentando dúvidas sobre o local $\mathrm{e}$ as formas de encontro, revelando que o apoio matricial entre esses serviços está em processo inicial. Em relação aos encaminhamentos, os profissionais da SF questionaram quais os critérios para encaminhar os pacientes ao CAPS e quais casos seriam discutidos nos encontros de matriciamento. O grupo refletiu sobre a importância da discussão dos casos, buscando auxiliar a SF na condução clínica.

Foi discutido o papel e a importância do CAPS III no acolhimento da crise. Porém, este estudo identificou a necessidade de aprofundar o debate sobre o papel da ESF no atendimento à crise, elucidando critérios de avaliação de risco, vulnerabilidade e urgência em saúde mental, a fim de que a APS avalie, acolha e aborde os pacientes, antes de encaminhá-los.

Sendo assim, identifica-se que os encontros do grupo misto permitiram a reflexão sobre a necessidade da responsabilização da ESF pela saúde mental e a importância da redução dos encaminhamentos indiscriminados. É fundamental que o apoio matricial seja colocado em pauta como uma ação estratégica na atenção em saúde mental, em ambos os serviços.

Dentre os caminhos possíveis para a realização do apoio matricial em saúde mental, destacaram-se a coordenação conjunta de grupos e das visitas domiciliares. Outras ações sinalizadas foram as 
discussões de casos clínicos complexos e as atividades de capacitação em serviço.

A coordenação conjunta de grupos foi identificada como uma das grandes necessidades da ESF. As falas apontaram que nos grupos há mais tempo para escuta das singularidades, mas há uma deficiência na formação dos profissionais da SF para abordar aspectos subjetivos e emocionais. O grupo de gestantes foi o mais citado, sendo destacados também os grupos de idosos e o de tabagismo.

Os grupos na SF consistem em ferramentas potentes para a mudança no modelo de atenção à saúde, entendidos como espaços coletivos para reflexão e para transformação de realidades. Além de auxiliar na organização da demanda, diminuindo os pedidos por consultas médicas, os grupos possuem especificidades, como, por exemplo, a possibilidade de "socialização, integração, apoio psíquico, trocas de experiências, trocas de saberes e construção de projetos coletivos. O pertencimento a um grupo favorece a saúde mental do sujeito" (Onocko Campos \& Gama, 2010, p. 241). Porém, muitas vezes na APS prevalece uma prática que degrada os grupos, sendo realizada uma espécie de consulta coletiva ou uma sessão informativa, buscando unicamente a transmissão de informações por parte dos profissionais, sem espaço para as trocas vitais e verdadeiras entre os participantes, o que gera a não-adesão dos usuários. Nesse sentido, formação e capacitação sobre o funcionamento dos grupos e um trabalho em parceria com os profissionais da saúde mental são considerados caminhos para qualificar a coordenação dos grupos pela ESF (Onocko Campos \& Gama, 2010).

Outro caminho sinalizado pelos participantes da pesquisa para as ações de matriciamento foi a realização conjunta de visitas domiciliares. Essa atividade é uma ação que já ocorre entre os dois serviços. Para isso, o ACS foi destacado como fundamental, por conhecer bem a comunidade. Destaca-se que a visita domiciliar, por ser uma atividade desenvolvida no território o mais próximo possível da casa das pessoas é uma ação preconizada tanto para os profissionais da ESF como para os do CAPS.

\section{Considerações finais}

Conclui-se que ambos os serviços investigados estão organizando atividades e implantando ações e, nesse contexto, o apoio matricial encontra-se em processo inicial, ocorre esporadicamente, quando alguma situação demandar.

Levando-se em consideração que a saúde mental não é contemplada na Política Nacional de Atenção
Básica como uma das áreas estratégicas de atuação no Brasil, que há escassos indicadores de saúde mental no sistema da informação nacional, a saúde mental ainda é uma área a ser fortalecida do processo de trabalho das equipes de APS. Assim, ao se investigar a implantação do apoio matricial em saúde mental, esse fato se fez presente nas equipes de $\mathrm{SF}$ pesquisadas.

Neste contexto, acredita-se que as equipes de SF e os gestores se preocupam primeiramente em organizar e implantar outras ações, deixando a saúde mental para momento posterior. Dessa forma, percebe-se que há carências na compreensão de que a saúde mental é um tema transversal, presente em todas as áreas, ou seja, de que a saúde mental está contemplada, por exemplo, na saúde da mulher, na saúde da criança, do homem.

Os resultados deste estudo apontaram para a dificuldade das equipes de SF na abordagem dos casos de saúde mental, que foram associadas à falta de capacitação na área e insuficiências na formação, gerando temor dos profissionais em relação às pessoas em sofrimento psíquico grave e insegurança no cuidado. Indicaram, também, preconceito e dificuldades na identificação das situações de saúde mental. Ainda, sobre as ações de saúde mental desenvolvidas na ESF, esta pesquisa sinalizou a coexistência da atenção asilar e da clínica ampliada nas práticas de cuidado das equipes.

Sendo assim, para o fortalecimento da saúde mental na APS, são fundamentais investimentos na educação permanente, como, por exemplo, o fortalecimento do apoio matricial, a realização de cursos de especialização, a oferta de cursos de atualização e de aperfeiçoamento, a ampliação de vagas para a residência multiprofissional ou médica. Embora esta já seja uma das preocupações da Coordenação Nacional de Saúde Mental, há necessidade de se ampliar esta oferta (Delgado, 2009).

Além disso, é importante, por parte da gestão, a priorização de indicadores e metas de saúde mental para a ESF. Destaca-se a necessidade de envidar esforços na qualificação dos sistemas de informação para o monitoramento e avaliação das ações de saúde mental e de apoio matricial desenvolvidas, possibilitando a construção de diagnósticos dos problemas de saúde mental e propostas de ações condizentes com as necessidades. Tais atitudes poderiam colocar a saúde mental em um lugar diferenciado para as equipes APS, e o apoio matricial, dessa forma, ser entendido pela ESF como uma ação necessária para a qualidade da atenção prestada à população.

Este estudo não previu a análise aprofundada da organização do trabalho da unidade de SF pesquisada, mas sinalizou que o processo de trabalho necessita ser 
discutido e revisado, em razão da sensação de sobrecarga identificada nos participantes da pesquisa. Ressalta-se que as condições de trabalho dessa unidade são boas, a unidade é bem preservada, equipada e abastecida. Um aspecto que pode contribuir para essa sensação de sobrecarga refere-se ao grande quantitativo de equipes de SF atuando no mesmo espaço físico, que na realidade estudada foi de mais de dez equipes. Estudos futuros que investiguem o processo de trabalho em unidades com mais de três equipes de SF podem contribuir para esta discussão.

No que se refere ao CAPS, a articulação com o território e a integração com outros serviços da rede são considerados fundamentais para a atenção psicossocial. Mas este estudo identificou um serviço com questões internas a serem debatidas, como, por exemplo, a necessidade de fortalecer o trabalho em equipe interdisciplinar, o que favorecerá o trabalho com o território.

Ressalta-se que o apoio matricial é uma diretriz nova para a saúde mental no Brasil, tendo cerca de uma década de existência, o que demanda investimentos em formação que fortaleça a interdisciplinaridade, como é o caso dos Programas de Educação pelo Trabalho Saúde/Saúde Mental e os Programas de Residência Multiprofissionais ou Médicas. O apoio matricial exige habilidades por parte dos profissionais no que se refere à abertura para a troca de saberes e para a construção conjunta de condutas clínicas, investimentos necessários para cursos de graduação, pós-graduação e aperfeiçoamento.

Acredita-se que esta pesquisa auxiliou os participantes a refletir sobre a importância do apoio matricial no cuidado em saúde mental e indicou caminhos possíveis a serem trilhados, levando em conta as necessidades da ESF e a realidade do CAPS III. Neste sentido, a adoção da pesquisa-ação como modalidade de investigação foi relevante para os participantes tomarem consciência conceitual e das suas necessidades e possibilidades de ação no que se refere à implantação do apoio matricial.

$\mathrm{O}$ apoio matricial busca romper com o modelo centrado nos especialismos, fortalecendo a atenção psicossocial, inclusive para as situações de crises. Este estudo evidenciou a necessidade de se pautar a discussão sobre o acolhimento e atendimento da crise na APS.

Por fim, ressalta-se o quão rica foi a experiência desta pesquisa: proporcionou a vivência de encontro com dois serviços implantados em uma realidade antes carente de atenção à saúde, possibilitou a aproximação de dois serviços em suas especificidades e realidades e permitiu encontros de integração entre equipes. Acima de tudo, possibilitou a análise da implantação de uma ação fundamental para o fortalecimento da atenção psicossocial em saúde mental.

Porém, várias foram as questões emergentes que merecem ser aprofundadas por estudos futuros, a fim de que ocorram avanços no entendimento da complexidade da atenção em saúde mental no Brasil. Dentre elas, destaca-se a necessidade de ampliar a discussão sobre o papel da APS na atenção psicossocial dos sujeitos em sofrimento psíquico, o papel da equipe interdisciplinar na clínica da reforma psiquiátrica, dentre outras. Espera-se que este trabalho contribua, em especial, para a reflexão sobre a implantação de ações de apoio matricial e possa subsidiar gestores e equipes na efetivação dessa prática.

\section{Referências}

Barbier, R. (2002). A pesquisa-ação. Brasília: Plano.

Bardin, L. (1977). Análise de conteúdo. Lisboa: Edições 70.

Brasil (2004). Ministério da Saúde. Saúde mental no SUS: os centros de atenção psicossocial. Brasilia: Secretaria de Atenção à Saúde. Departamento de Ações Programáticas Estratégicas.

Brasil (2005). Ministério da Saúde. Reforma psiquiátrica e politica de saúde mental no Brasil. Brasília: Secretaria de Atenção à Saúde. Departamento de Ações Programáticas e Estratégicas em Saúde. Coordenação Geral de Saúde Mental. OPAS.

Brasil (2009). Ministério da Saúde. Clínica ampliada e compartilhada. Brasília: Secretaria de Atenção à Saúde. Política Nacional de Humanização da Atenção e Gestão do SUS.

Brasil (2010). Ministério da Saúde. Diretrizes do NASF: Núcleo de Apoio à Saúde da Família. Brasília: Secretaria de Atenção à Saúde. Departamento de Atenção Básica.

Campos, G. W. S. (1999). Equipes de referência e apoio especializado matricial: um ensaio sobre a reorganização do trabalho em saúde. Ciência \& Saúde Coletiva, 4(2), 393-403. doi:10.1590/S141381231999000200013.

Campos, G. W. S., \& Guerreiro, A. V. P. (2010). Manual de práticas de atenção básica: saúde ampliada e compartilhada. São Paulo: Hucitec.

Costa-Rosa, A. (2000). O modo psicossocial: um paradigma das práticas substitutivas ao modo asilar. Em P. Amarante (Org). Ensaios: subjetividade, saúde mental, sociedade (pp. 141-168). Rio de Janeiro: Fiocruz.

Delgado, P. G. G. (2009). Formação em saúde mental para o SUS: a estrada pedregosa e a máquina do mundo. Em I. I Costa, \& T. M. Grigolo (Orgs). 
Tecendo redes em saúde mental no cerrado (pp. 13-15). Brasília: ABRAFIPP/UnB/MS.

Franco, M. L. P. B. (2008). Análise de conteúdo. Brasília: Liber.

Guljor, A. P. F. (2003). Os centros de atenção psicossocial: um estudo sobre a transformação do modelo assistencial em saúde mental (Dissertação de Mestrado). Escola Nacional de Saúde Pública, Fundação Oswaldo Cruz, Rio de Janeiro, Brasil.

Onocko Campos, R.T., \& Gama, C. (2010). Saúde Mental na Atenção Básica. Em G. W. S. Campos, \& A. V. P. Guerreiro (Orgs). Manual de práticas de atenção básica: saúde ampliada e compartilhada. São Paulo: Hucitec.
Portaria no 2.488/2011 de 21 de outubro. Diário Oficial da União - Seção 1. Ministério da Saúde. Brasília, Distrito Federal, Brasil.

Rio de Janeiro (2009). Contrato de gestão. Secretaria Municipal da Saúde e Defesa Civil e Viva Comunidade. Rio de Janeiro.

Starfield, B. (1994). Is primary-care essential? Lancet, 8930(344), 1129-1133.

Thiollent, M. (1996). Metodologia da pesquisa-ação ( $7^{\mathrm{a}}$ ed.). São Paulo: Cortez.

Recebido em: 08/10/2012

Reformulado em: 26/02/2013

Aprovado em: 14/03/2013

Sobre os autores:

Fabiane Minozzo é mestre em Psicologia Clínica e Cultura (UnB), com especialização em Saúde Mental (UnB) e residência em Atenção Primária à Saúde (ESP/RS). Trabalha na gestão da Saúde da Família e Saúde Mental, no município do Rio de Janeiro.

Ileno Izídio da Costa é doutor em Psicologia Clínica (UnB/Warwick), coordenador de Extensão do Instituto de Psicologia da Universidade de Brasília (Unb), professor adjunto do Departamento de Psicologia Clínica (UnB), coordenador do Centro de Atendimento e Estudos Psicológicos, do Grupo Personna (Estudos e pesquisas sobre violência, criminalidade e perversão) e do Grupo de Intervenção Precoce nas Psicoses (UnB), e presidente da Associação de Saúde Mental do Cerrado.

Contato com os autores:

Rua Antônio Parreiras, 25, apartamento 606 - CEP: 22411-020 - Rio de Janeiro/RJ, Brasil.

E-mail: fabiminozzo@yahoo.com.br

Houve a correção na ordem dos autores.

Publicado:

Ileno Irídio da Costa - Universidade de Brasilia, Brasilia-DF, Brasil

Fabiane Minozzo - Universidade de Brasilia, Brasilia-DF, Brasil

Correto:

Fabiane Minozzo - Universidade de Brasilia, Brasilia-DF, Brasil

Ileno Irídio da Costa - Universidade de Brasilia, Brasilia-DF, Brasil

Psico-USF, Bragança Paulista, v. 18, n. 1, p. 151-160, jan./ abril 2013 
